(Fig. 1b) a 1-megohm potentiometer replaces the two $10 \mathrm{~K}$ resistors with the center tap connected to the bias voltage clamping circuit. The potentiometer allows the adjustment of the bias vol tage to the modulator tubes.

While the tubes are resting in either the steady ON state or steady OFF state, the bias voltages are equal regardless of the potentiometer setting since virtually no current is passing through the potentiometer. During the transient portions of the switching operation when the bias voltages on the tubes are changing, there is a slight current and the difference in resistance to the two channels, as determined by the potentiometer setting, will have the effect of leading one tube ahead of the other.

The potentiometer can also be inserted in place of the two resistors R 114 and R 115 (see Grason-Stadler operating manual), in the B switching circuits. With the two potentiometers the two channels can be brought into close coordination. If the modulator tubes are sufficiently similar in characteristics the two channels can be made to switch virtually simultaneously. If the tubes are not sufficiently similar to allow elimination of the onset and offset differences, the potentiometers can be adjusted so that onset difference is nil and the offset difference is at a minimum.

Another modification can be made to adjust for simultaneous switching of two switches that are triggered by the same timer. The $30 \mathrm{~K}$ resistor (R27) that determines the plate voltage on $V_{4}$ and $V_{5}$ can be replaced with a $22 \mathrm{~K}$ resistor and a $10 \mathrm{~K}$ potentiometer as shown in Fig. 2. This procedure allows for regulation of the voltage of the clamping circuit, hence, the time at which the modulator tubes will be turned on. The best way to check for differences in switching onset and offset disparities is by a differential amplifier on an oscilloscope. A low remainder voltage indicates a balanced system.

The potentiometers can be mounted on the rear of the chassis behind the Channel 1 switching section.

\section{NOTE}

1. This study was supported by NSF Grant GB-6815 and NIH Grant MH 11218.
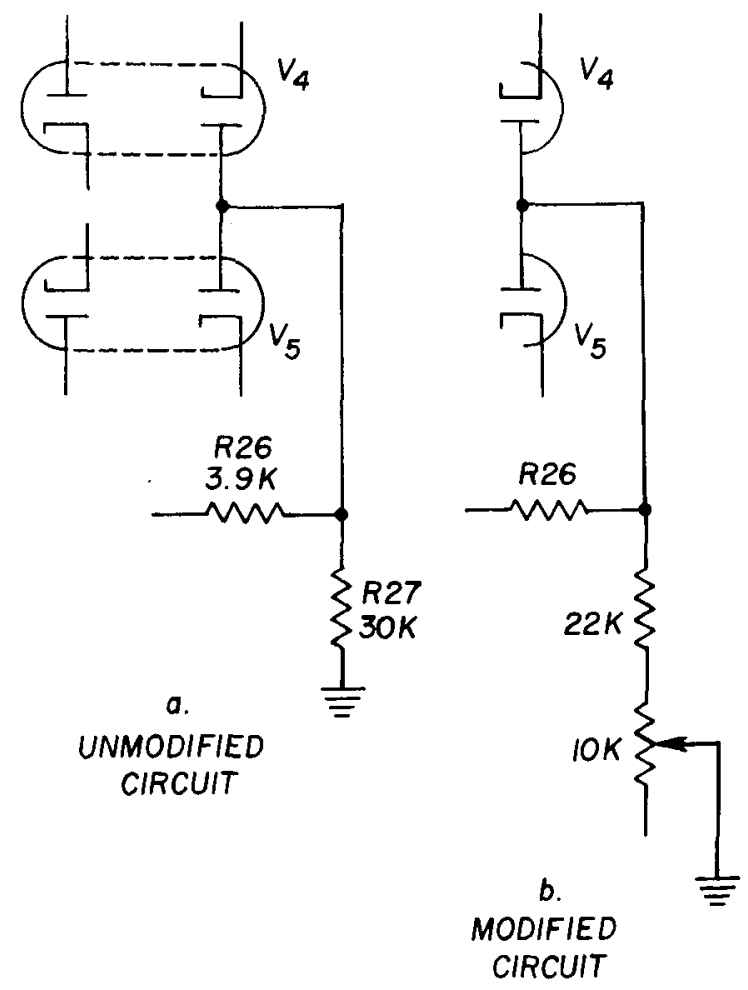

Fig. 2. Schematic for adjusting overall switching tube bias.

\title{
A simple method of producing discrete subcortical lesions using radio-frequency currents ${ }^{1,2}$
}

\author{
T. E. LeVERE and LAWRENCE R. NICHOLSON, NORTH \\ CAROLINA STATE UNIVERSITY, Raleigh, North Carolina \\ 27607
}

The described procedures represent an evaluation of the use of radio frequency currents in the production of discrete subcortical brain lesions. The methods described and the electrodes used indicated that RF lesion size was closely and linearly related to a simple $V d c$ index and that the resultant lesions were equally distributed around the position of the lesion electrode with extremely smooth and consistent perineters.

The utility of a reliable method for destroying discrete areas of neural tissue below the surface of the brain can be estimated from the varied techniques and equipment that have been devised for such operative procedures. By and large, the use of unidirectional current concentrated at the tip of an electrode stereotaxically located within the brain has enjoyed the most success. This electrolytic technique requires simple equipment and provides close correlation of lesion size with millicoulomb flow (Macintyre et al, 1957). The production of subcortical brain lesions, however, with dc currents is not without its drawbacks. Perhaps the most serious effects are the inconsistency of lesion shape and the deposition of metallic ions at the perimeter of the insult, with the subsequent possibility of stimulation of adjacent brain areas (Reynolds, 1963).

An alternative technique for producing subcortical lesions utilizes bidirectional radio-frequency $(\mathrm{RF})$ currents that, when concentrated at the tip of an electrode, can cause neural destruction by the conversion of electrical energy into heat (Aronow, 1960; Reynolds, 1963). This method has been used in our laboratory and the results are described herein. Specifically, lesion size was studied as a function of electrode configuration and power output.

\section{SUBJECTS}

The Ss were four male and nine female adult Long-Evans hooded rats from the colony maintained at North Carolina State University. All Ss were in good health and treated in accord with accepted practices concerning experimental animal surgery.

\section{APPARATUS}

A Grass Model LM-3 RF lesion generator was used for all experimental lesions. The electrodes were constructed from either 
(1) 28-g stainless steel suture wire completely insulated except for the cut end cross section or insulated to within $1 \mathrm{~mm}$ of the end, or (2) stainless steel insect pins (size 00$)^{3}$ insulated to with in $1 \mathrm{~mm}$ or $2 \mathrm{~mm}$ of the pointed end. Thermobond $\mathrm{M}-472$ was used as insulation on all electrodes. 4

Two methods of measuring and setting power output of the LM-3 were used. The first employed a cathode ray oscilloscope connected to the output terminals leading to the $S$ and provided a direct measure of the current delivered to the animal. The second method utilized a voltmeter set to the $0-5 \mathrm{~V}$ dc scale. The meter was connected to the monitoring terminals provided on the front panel of the lesion generator. Due to the conversion of ac currect to $\mathrm{dc}$ via a rectifier, diode, and capacitor circuit, the reading was not linearly related to the output delivered to the $\mathrm{S}$. It did, however, provide a reliable index of output current and was considered the method of choice in view of its simplicity in relation to the surgical procedures. The data provided here are based upon this second method of monitoring current output.

All wire connections between the $S$ and the lesion generator were standard Beldon hookup (22 AWE, 7 × 30 Type MW) wire. The wire leads to the anal electrode and the lesion electrode were $30 \mathrm{~cm}$ in length and physically separated to prevent capacitance coupling. The brass anal electrode measured $5 \mathrm{~cm}$ in length and $6 \mathrm{~mm}$ in diam and was coated with EKGsol ${ }^{5}$ to insure electrical contact. Connections to the voltmeter were standard zip cord.

\section{PROCEDURE}

Each $S$ was prepared for surgery with a preoperative intraperitoneal injection of $0.2 \mathrm{cc}$ of atropine sulfate, followed 10-15 min later with an injection of sodium pentobarbital $(60 \mathrm{mg} / \mathrm{kg}$, IP). The anesthetized $S$ was then placed in a stereotaxic instrument and the head positioned according to DeGroot (1963). Following retraction of the skin, the positions of the electrode placements were marked on the skull and all positions drilled with a dental burr. A single electrode was then lowered through one of the holes into the selected subcortical nucleus (anterior area of hypothalamus; dorsomedial nucleus of hypothalamus; lateral septal nucleus or dorsomedial nucleus of thalamus), according to the coordinates given by DeGroot (1963), with the lead wire fastened to the electrode and its carrier to minimize electrode movement once the electrode was positioned within the brain. The electrode lead wire and the lead wire from the previously positioned anal electrode were then attached to the lesion generator. When all connections were made and checked, the timer on the lesion generator was set (20 or $30 \mathrm{sec}$ ) and the "intensity" dial advanced to a position slightly below that required to attain the desired $\mathrm{V} d c$ current indication for the particular lesion being performed. The lesion was initiated and the final adjustment of the "intensity" dial made to bring the current indication to the desired level. This final adjustment could always be carried out $2-3 \mathrm{sec}$ after initiation of the lesion. When the lesion timer completed its cycle, the electrode was withdrawn and discarded. The particular intensity setting required to attain the desired current flow was recorded along with a determination of whether or not the current was maintained for the specified time (i.e., did the lesion run its course). Other lesions were performed at the remaining subcortical locations in accord with the above procedures but always with a fresh electrode.

In order to obtain valid estimates of tissue damage, each animal was immediately perfused following the operative procedure with saline and $10 \%$ formalin. While it is realized that this procedure might introduce a certain amount of error since some of the affected cells would not indicate complete destruction until some time following the insult, it was decided that this error would be far less than that introduced by the mechanical, but functionally insignficant, readjustment of neural tissue that occurs when Ss are permitted several weeks of postoperative survival. Recent research has, however, suggested that this precaution was unnecessary since there is little difference in size of sequentially produced posterior hypothalamic RF lesions that are, respectively, 3 and 13 days old at the time of perfusion. 6

Following perfusion, each brain was removed from the skull and placed in 10\% formalin for at least $24 \mathrm{~h}$. The brain was then transferred to a $1: 1$ mixture of $10 \%$ formalin and $20 \%$ alcohol, and after at least one additional 24-h period, it was frozen and sectioned at 40 micra with every fifth section through the lesion area mounted on slides. The mounted sections were then either photographed in the "raw" state or stained with cresyl violet. Lesion size was determined by measuring the largest horizontal diameter from the enlarged photographs or from the projected images of the stained sections. All size estimates were made to the nearest $0.1 \mathrm{~mm}$.

\section{RESULTS}

Figure 1 illustrates the general configuration of the lesions obtained with the described procedures while Fig. 2 relates lesion size to power output as indexed by the dc voltage measured across the monitoring terminals provided with the Grass LM-3. The figure excludes from consideration those lesions attempted with electrodes having only the cross section of the wire exposed and those lesions attempted with 2-mm tip exposures. These results were not included because the small tip area did not produce any noticeable neural destruction, and the increased size of the 2-mm tip, although producing a somewhat larger lesion, was insensitive to our measures of lesion size because of the distribution of RF power at the intensity settings employed. That is, with a $1 \cdot \mathrm{mm}$ tip, an increase of $0.05 \mathrm{~V}$ dc produced a twofold increase in lesion diameter while the same increase in power when distributed over a $2-\mathrm{mm}$ tipped electrode failed to produce any increase in horizontal lesion size and all lesions were approximately football-shaped with $2.0 \mathrm{~mm}$ diam. However, lesions produced with comparable electrode configurations were directly related to monitored current output up to approximately $0.30 \mathrm{~V} \mathrm{dc}$ after which the probability of maintaining current continuity between the lesion electrode and the indifferent ground electrode rapidly decreased until approximately $0.45 \cdot 0.50+\mathrm{V}$ dc. At this level no lesion could be completed and size estimates were of little relevance. Note that the two current

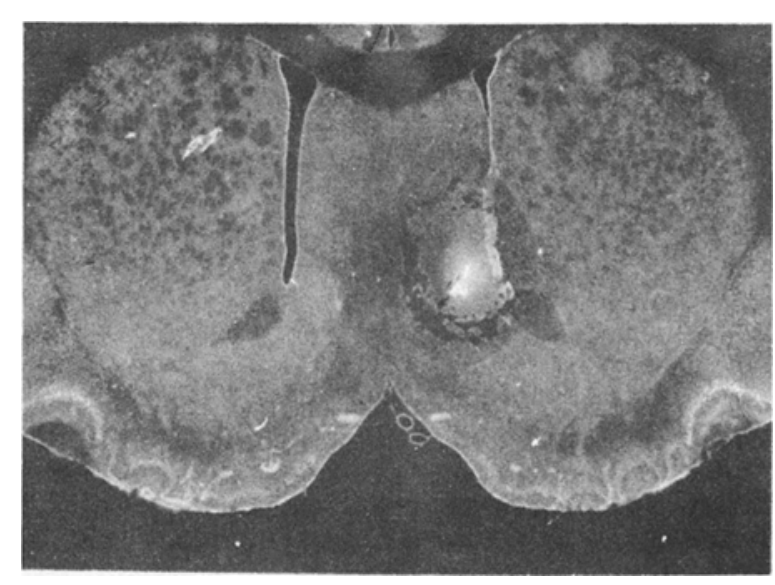

Fig. 1. The general configuration of the RF lesions produced by 00 insect pins insulated to within $1 \mathrm{~mm}$ of the tip. 


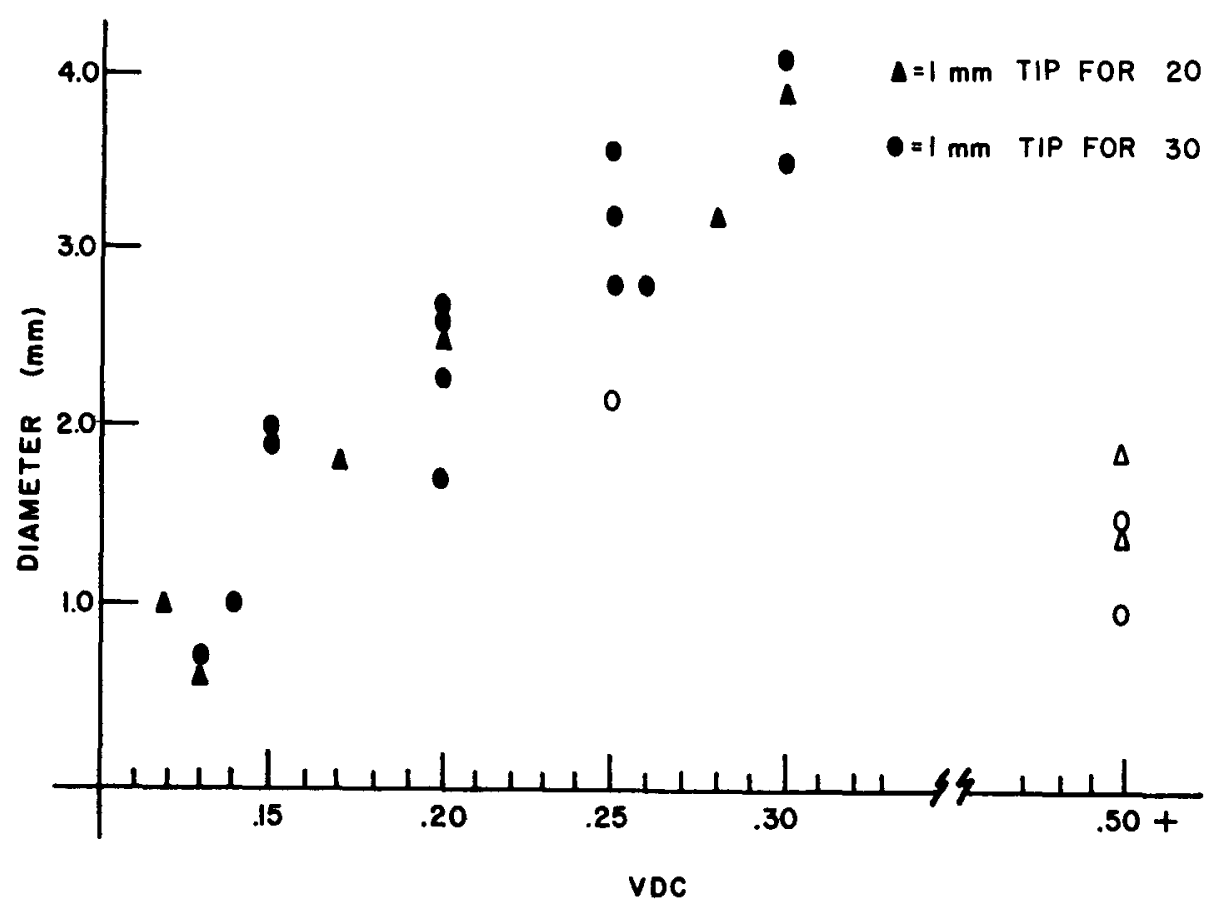

Fig. 2. The relation of horizontal lesion diameter to dc voltage as monitored from the terminal provided on the Grass LM-3. Filled points indicate completed lesions; open points represent incompleted lesions.

durations ( 20 and $30 \mathrm{sec}$ ) appeared to be relatively insignificant, and lesion size appeared primarily related to current intensity.

\section{DISCUSSION}

A physical requirement for the production of either electrolytic or RF lesions is the maintenance of electrical continuity between the lesion electrode and the indifferent ground electrode. The problem is to generate sufficient heat to cause neural destruction but not enough to cause the tissue to boil and produce a gas bubble around the electrode tip that will break electrical continuity. A primary consideration related to the conversion of electrical energy to heat is the area over which the RF current is distributed for any given power setting; within limits, the larger the electrode tip area, the greater can be the power applied before the tissue temperature reaches the boiling point. In the present study, electrodes that were constructed from 28-g stainless steel surgical wire, with only the cross sectional area of the wire exposed, failed to produce any neural destruction at any of the power settings. This was probably due to the concentration of RF current over such a small area that it caused the tissue to boil and form a gas bubble at the instant the lesion was initiated. This then broke electrical continuity before enough destructive heat could be generated over any expanse of neural tissue and consequently spared all neural tissue except that affected by lowering the electrode itself. Alternatively, similar power settings delivered to a 1-or 2-mm tipped electrode will generate sufficient heat to produce neural destruction but still remain below the boiling point. However, 1- or 2-mm tip sizes may not be appropriate for all power settings. It is possible even with these relatively large areas to cause tissue to boil provided sufficient current is applied over a short enough period of time. This is not only undesirable because of a loss of control over lesion size (the lesion does not go to completion), but also because the physical configuration of the lesion exhibits extremely ragged edges as opposed to a smooth, discrete outline produced when boiling is avoided.

By and large, radio frequency current appears to offer a precise method of producing discrete subcortical lesions of predetermined size. The method has the advantage of producing quite smooth lesion perimeters with the electrode track consistently bisecting the lesion area and is simple and easily adapted to standard operative procedures.

\section{REFERENCES}

ARNOW, $S$. The use of radio-frequency power in making lesions in the brain. Journal of Neurosurgery, 1960, 27, 431.

DeGROOT, $\mathbf{J}$. The rat forebrain in stereotaxic coordinates. Amsterdam: Drukkerij Holland N.V., Second Printing, 1963.

MacINTYRE, W. J., BIDDER, T. G., \& ROWLAND, V. The production of brain lesions with electric currents. In: Proceedings of the First National Biophysics Conference. Columbus, Ohio, 1957.

REYNOLDS, R. W. Ventromedial hypothalamic lesions without hyperphagia. American Journal of Physiology, 1963, 204, 60.

\section{NOTES}

1. This investigation was supported by Research Grant No. MH 15131-01 from the National Institute of Mental Health to T. E. LeVere.

2. Reprint requests should be sent to the attention of T. E. LeVere.

3. Available from Clay Adams, Parsippany, New Jersey.

4. Available from Sterling Varnish Company, Haysville Boro, Sewickley, Pennsylvania. Each electrode was given five coats of varnish and baked at $300 \operatorname{deg} \mathrm{F}$ for at least $4 \mathrm{~h}$ between each coat.

5. Available from Burton, Parsons \& Company, Washington, D.C.

6. LeVere, T, E. The inability of the posterior hypothalamic area to mediate endogenous functional reorganization. Unpublished manuscript, 1969. 